\title{
Force Feedback Control of Tele-Operation Based on Electro-Hydraulic Multi-Freedom Manipulator \\ Ming-De GONGa ${ }^{a}$ Kai-Wen CHEN ${ }^{\mathrm{b}, *}$ and Tao $\mathrm{NI}^{\mathrm{c}}$
}

${ }^{1}$ School of Mechanical Science and Engineering, Jilin University, 130025 Changchun, China

agmd@jlu.edu.cn, bchenkw15@mails.jlu.edu.cn, cnitao@jlu.edu.cn

${ }^{*}$ Corresponding author: Kai-wen CHEN

Keywords: Tele-operation, Position space, Control strategy, Bilateral servo control.

\begin{abstract}
The paper presents the control strategy to solve the problems that multiple-freedom force and displacement coupling existing in the bilateral servo force-feedback control architecture for tele-operation parallel manipulators. The control strategy transfers the values of the working resistance connected to the slave manipulator and its direction to the master side through the position space, which provides the operator with the constrained space the same as the slave side. The experimental results show that the proposed force-feedback control strategy is effective and markedly improves the manipulability for the master-slave control system.
\end{abstract}

\section{Introduction}

When operating the tele-robot remotely, the operator not only has to be provided with visual information, but also needs to know the working resistance and the force sensing caused by obstacle interference [1-4].

Designing the control strategy of the bilateral servo force-feedback control system is a key link in designing tele-operation system $[5,6]$.

The paper proposes a novel force-feedback control strategy from the aspect of position space, where the control lever in the master side provides the same position space with slave side interference. Therefore, the operator would have better perception and master of the key elements in the slave-side interference force, which realizes the bilateral servo force-feedback control.

\section{Bilateral Servo Force-Feedback Control Strategy}

The paper proposed the force feedback strategy from the aspect of position space, where the master side lever offers the same position space with the slave side interference. The structure of the novel force-feedback strategy is illustrated in figure 1.

In the usual force-feedback strategies [7], the feedback force on the lever is generated by the slave- side testing force and the deviation between the master and the slave position, which is illustrated in the formula (1) $\mathrm{Km}$ is the gain coefficient, and the direction of the force feedback to the master side is decided by the acting direction of $\mathrm{X}_{\mathrm{m}}$ and $\mathrm{X}_{\mathrm{s}}$. Formula (1) discussed the feedback force from the aspect of master-slave position tracing, however the real situation is that the direction of $\mathrm{X}_{\mathrm{s}}$ is not exclusively related to the moving direction of slave side manipulator, but also partly decided by the direction of obstacle interference in the surroundings.

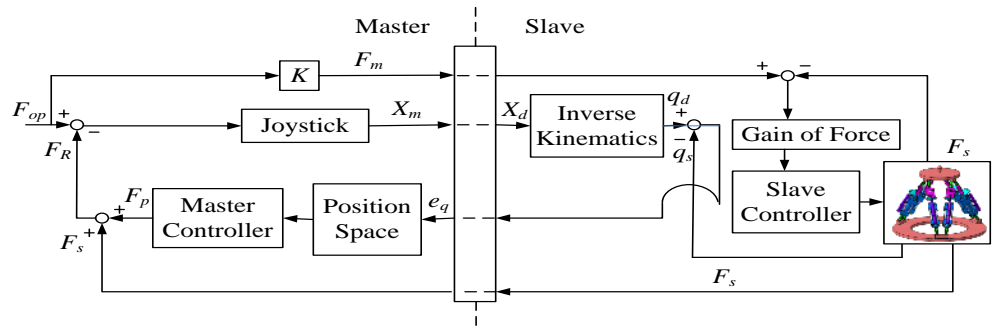

Figure 1. Structure of the force-feedback control architecture. 
That is the reason why the operator in the master side couldn't have an accurate perception of the interference force direction. It misleads the operator with the wrong interference force and displacement information of the slave side manipulator, and sometimes even fails to fulfil the anticipated operation, especially in the cases with multi-freedom master-slave manipulators, interference force and strong displacement coupling. The research adopted the control strategy in figure 1, maintaining the generality, with the six DOF operation space in the slave side. The feedback force of lever satisfy formula (2), where $\boldsymbol{q}_{\boldsymbol{d}}$ and $\boldsymbol{q}_{\boldsymbol{s}}$ are respectively the expected joint angular region of the slave side lever with the manipulate force and the actual joint region that the slave manipulator reached. $\mathbf{R}_{\mathbf{m}}$ is the external constraint spatial matrix. The transposed determinant of Jacobean determinant is the force mapping from the element message of the constraint force in the operation space to the joint space. Formula (2) and (3) transferred the mapping to the generalized constraints and present them in the freedoms of the master and slave sides.

$$
\begin{gathered}
F_{R}=K_{m}\left(X_{m}-X_{s}\right)+F_{s} \\
\left(F_{R}\right)_{6 \times 1}=\left(R_{m}\right)_{6 \times 6} \cdot\left(q_{d}-q_{s}\right)_{6 \times 1}+\left(F_{s}\right)_{6 \times 1} \\
R_{m}=\left[\Phi\left(J_{2}, J_{3}, \ldots J_{6}\right)^{\perp}, \Phi\left(J_{1}, J_{3}, \ldots J_{6}\right)^{\perp},\right. \\
\left.\ldots . ., \Phi\left(J_{1}, J_{2}, \ldots J_{5}\right)^{\perp}\right]
\end{gathered}
$$

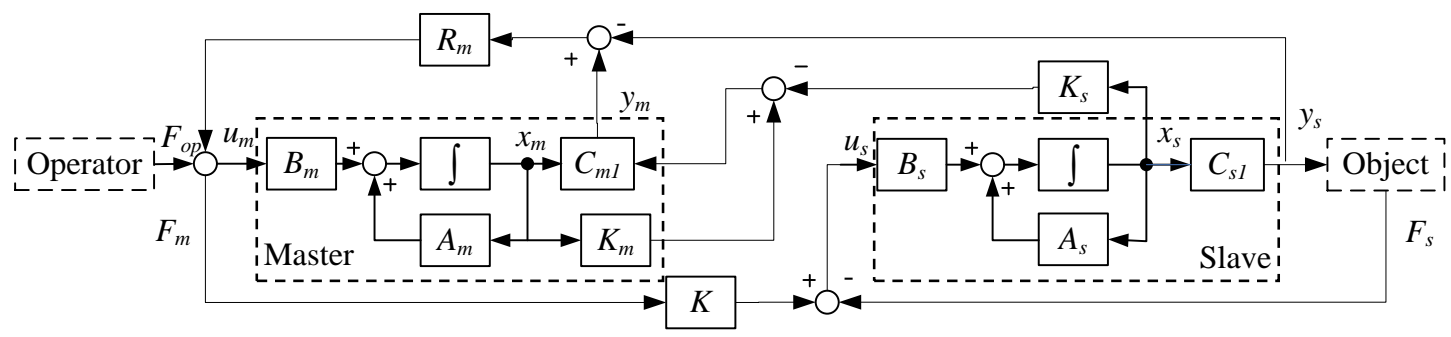

Figure 2. State space model of master-slave tele-robot.

\section{The Stable Condition of the Force-Feedback Control Strategy}

The state space model of master-slave tele-robot is given below in Fig. 2, which includes the operator, the main manipulator, the slave manipulator and the surroundings. $F_{m}$ denotes the manipulating force applied to the master side lever (usually considered equivalent to $F_{o}$ ) while um and us respectively represents the control signal of the master side and the slave side. $x_{m}$ and $x_{s}$ are respectively the state vector of the master and the slave side. $y_{m}$ and $y_{s}$ are respectively the output of the master side and the slave side.

The state spaces can be represented by the equations below

$$
\left\{\begin{array}{l}
\dot{x}(t)=\boldsymbol{A x}(t)+B u(t) \\
\boldsymbol{y}(t)=\boldsymbol{C x}(t)
\end{array}\right.
$$

Where: $\boldsymbol{x}(t)=\left(\begin{array}{ll}x_{m} & x_{s}\end{array}\right)^{T}, \boldsymbol{y}(t)=\left(\begin{array}{ll}y_{m} & y_{s}\end{array}\right)^{T}, \boldsymbol{A}=\left(\begin{array}{ll}A_{m} & A_{s}\end{array}\right)^{T}, \boldsymbol{B}=\left(\begin{array}{ll}B_{m} & B_{s}\end{array}\right)^{T}$.

With Lyapunov function chosen as

$$
A^{\prime} P+P A=-Q
$$

For the time-delayed systems, there is

$$
\dot{\boldsymbol{x}}(t)=\boldsymbol{A} \boldsymbol{x}(t)+\boldsymbol{B} \boldsymbol{x}(t-\tau)
$$

Choose the quadratic form as the Lyapunov function 


$$
\begin{aligned}
& V=x^{\prime}(t) P x(t)+x^{\prime}(t) \int_{-\tau} P_{1}(s) x(t+s) d s+x(t) . \\
& \int_{-\tau}^{0} x^{\prime}(t+s) \boldsymbol{P}_{1}^{\prime}(s) d s+\int_{-\tau}^{0} \int_{-\tau}^{0} x^{\prime}(t+s) \boldsymbol{P}_{2}^{\prime}(s) x(t+\tau) d s d \tau \\
& \dot{x}=\left[\begin{array}{cccc}
0 & 1 & 0 & 0 \\
-\frac{k_{m}}{m_{m}} & -\frac{b_{w}+b_{c}}{m_{m}} & 0 & 0 \\
0 & 0 & 0 & 1 \\
0 & 0 & -\frac{k_{s}+k_{c}}{m_{s}+m_{e}} & -\frac{b_{s}+b_{e}+b_{c}}{m_{s}+m_{e}}
\end{array}\right] \boldsymbol{x} \\
& +\left[\begin{array}{cccc}
0 & 0 & 0 & 0 \\
0 & 0 & 0 & \frac{b_{c}}{m_{m}} \\
0 & 0 & 0 & 0 \\
0 & \frac{b_{c}}{m_{s}+m_{c}} & 0 & 0
\end{array}\right] x(t-\tau)
\end{aligned}
$$

Choosing proper $\mathrm{P}, \mathrm{P}_{1}, \mathrm{P}_{2}$ decreased the total differential of $V$ below zero, which ensures the stability, therefore, simple $V$ functions are usually chosen in the real designing. In the state of teleoperation, the motion of the slave side manipulator can be divided to the constraint and the free motion. The paper only discussed the stability of the slave side motion with constraint.

Setting $\boldsymbol{x}=\left[\begin{array}{llll}x_{m} & \dot{x}_{m} & x_{s} & \dot{x}_{s}\end{array}\right]^{T}$, the manipulation force of human operation could be considered as the input of the force reflecting tele-operation system

$$
\dot{x}(t)=A_{1} x(t)+A_{2} x(t-\tau)
$$

Where:

$$
\begin{gathered}
\boldsymbol{A}_{1}=\left[\begin{array}{cc}
A_{11} & 0 \\
0 & A_{12}
\end{array}\right], \boldsymbol{A}_{2}=\left[\begin{array}{cc}
0 & A_{21} \\
A_{22} & 0
\end{array}\right], A_{11}=\left[\begin{array}{cc}
0 & 1 \\
-\frac{k_{m}}{m_{m}} & -\frac{b_{m}+b_{c}}{m_{m}}
\end{array}\right], \boldsymbol{A}_{12}=\left[\begin{array}{cc}
0 & 1 \\
-\frac{k_{s}+k_{c}}{m_{s}+m_{c}} & -\frac{b_{s}+b_{e}+b_{c}}{m_{s}+m_{c}}
\end{array}\right], \boldsymbol{A}_{21}=\left[\begin{array}{ll}
0 & 0 \\
0 & \frac{b_{c}}{m_{m}}
\end{array}\right], \\
\boldsymbol{A}_{22}=\left[\begin{array}{cc}
0 & 0 \\
0 & \frac{b_{c}}{m_{s}+m_{c}}
\end{array}\right] .
\end{gathered}
$$

Discuss the stability of system (4) with Lyapunov stability analysis, which means analysing by establishing Lyapunov equations

$$
V(x, t)=x(t)^{T} x(t)+\frac{1}{1-\gamma} \int_{-\tau}^{0} x(\theta)^{T} x(\theta) d \theta \geq 0
$$

where: $i \leq \gamma \leq 1$, establishing differential equations of (4) along the direction of system (5)

$$
\dot{V}(x, t)=2 \boldsymbol{x}^{T}\left(\boldsymbol{A}_{1} \boldsymbol{x}+\boldsymbol{A}_{2} \boldsymbol{x}(t-\tau)\right)+\frac{1}{1-\gamma} \boldsymbol{x}(t-\tau)^{T} \boldsymbol{x}(t-\tau)
$$

From $2 \boldsymbol{x}^{T} \boldsymbol{A}_{2} \boldsymbol{x}(t-\tau) \leq \boldsymbol{x}^{T} \boldsymbol{A}_{2} \boldsymbol{A}_{2}^{T} \boldsymbol{x}+\boldsymbol{x}(t-\tau)^{T} \boldsymbol{x}(t-\tau)$, equation (8) can be transformed to 


$$
\begin{aligned}
\dot{V}(x, t) & \leq x^{T}\left(2 A_{1}+A_{2} A_{2}{ }^{T}+\frac{1}{1-\gamma} I\right) x \\
& +x\left(t-\tau\left[I-\frac{1-\dot{t}}{1-\gamma} I\right]\right) x(t-\tau)
\end{aligned}
$$

If $\dot{V}(x, t)<0$, system (4) is asymptotically stable, and for equation (6), the right side of the inequality is :

$$
S=2 A_{1}+A_{2} A_{2}{ }^{T}+\frac{1}{1-\gamma} I<0
$$

The solution of equation (9) is the characteristic roots of matrix S, each having negative real part, namely $\operatorname{Re} \lambda_{i}(S)<0$

$$
S=\left[\begin{array}{cc}
2 A_{11}+A_{21} A_{21}^{\prime}+\frac{1}{1-\gamma} I & 0 \\
0 & 2 A_{12}+A_{22} A_{22}^{\prime}+\frac{1}{1-\gamma} \boldsymbol{I}
\end{array}\right]
$$

According to formula (11), $\mathrm{S}$ is a diagonal matrix. From (10), we have:

$$
\begin{array}{r}
S_{1}=2 A_{1 I}+A_{21} A_{2 I}^{\prime}+\frac{1}{1-\gamma} I<0 \\
S_{2}=2 A_{12}+A_{22} A_{22}^{\prime}+\frac{1}{1-\gamma} I<0
\end{array}
$$

Characteristic roots of $S_{\boldsymbol{I}}$ can be obtained from the characteristic polynomial $\operatorname{det}\left(\boldsymbol{S}_{1}-s \boldsymbol{I}\right)=s^{2}+a s+b$

Where:

$$
\left\{\begin{array}{l}
a=2 \frac{b_{m}+b_{c}}{m_{m}}-\frac{b_{c}{ }^{2}}{m_{m}{ }^{2}}-\frac{2}{1-\gamma} \\
b=\frac{1}{(1-\gamma)^{2}}+4 \frac{k_{m}}{m_{m}}+\frac{1}{1-\gamma}\left(\frac{b_{c}{ }^{2}}{m_{c}{ }^{2}}-2 \frac{b_{m}+b_{c}}{m_{m}}\right)
\end{array}\right.
$$

Thus we have

$$
\operatorname{Re} \lambda_{i}\left(S_{1}\right)<0 \Leftrightarrow\left\{\begin{array}{l}
a>0 \\
b>0
\end{array}\right.
$$

And the condition satisfying equation (13).

$$
\left\{\begin{array}{l}
2 \frac{b_{m}+b_{c}+b_{e}}{m_{s}+m_{e}}-\frac{b_{c}{ }^{2}}{\left(m_{s}+m_{e}\right)^{2}}-\frac{2}{1-\gamma}>0 \\
\frac{1}{(1-\gamma)^{2}}+4 \frac{k_{s}+k_{e}}{m_{s}+m_{e}}+\frac{1}{1-\gamma}\left(\frac{b_{c}{ }^{2}}{\left(m_{s}+m_{e}\right)^{2}}-2 \frac{b_{m}+b_{c}+b_{e}}{m_{s}+m_{e}}\right)>0
\end{array}\right.
$$

The above is the stable condition of the force telepresence system, namely $m_{m}, m_{s}, m_{e}, b_{m}, b_{s}, b_{e}$, $b_{c}$ meet the requirement of formula (13) (14) and (15). Therefore we conclude that the system remains stable as long as $b_{c}, m_{c}, b_{e}, k_{c}, k_{e}$ meet the requirement of the three equations when there are certain $m_{m}, m_{s}, m_{e}, b_{m}, b_{s}, k_{e}, k_{c}$. For any given $m_{e}, b_{e}, k_{e}$, the data area of $b_{c}, k_{c}$ can be obtained, by which we can design the controller. 


\section{Experiments and Result Analysis}

The lever adopted the Stewart parallel mechanism as the main structure of the 6-DOF lever, and consisted of a fixed platform (the lower base), a mobile platform (the upper base) and six branches. The upper pedestal and hydraulic control lever, along with the lower pedestal and hydraulic cylinder are both connected by hook joints, which combined with rotational freedom of the hydraulic cylinder rod, have realized the spherical coupling characteristics. By setting pull-press sensors between the hydraulic cylinder rod and hook joint, we are able to take measures of the output force or the force applied to the cylinder. Displacement sensor between the cylinder and the piston rod is used to measure the extension length of the piston rod.

During the experiment, according to the real time positions measured, the force signals and the relevant control parameters generated by the control strategy, the control of the master and the slave side levers are realized. Due to the six freedoms of the system, displacement curves of the six hydraulic cylinders or the tracing curves of the six freedoms are needed in order to describe the motions clearly. However, the paper mainly focused on the operation performances of the system, and the performance of the system is described with the experiment curves of two representative freedoms. The experiment set a rigid object on the front end of the third cylinder of the slave-side lever, in order to hinder the motion along the direction of the freedom, and furthermore simulated the feedback effects when contacting the rigid object. The experiment curves are shown in Fig.3 (ad).

The figure shows the force and position signals of the master and slave side cylinders with the novel force-feedback control strategy. The situations of the first and third cylinders are shown in the figure. When applying forces on the master-side lever, there is a difference in the force on the master and slave side. The motion of the slave side lever is generated before that of the master side, and the master-side lever therefore followed the movement of the slave-side lever, which determined the advance of the slave side lever to the master side lever in motion phases.
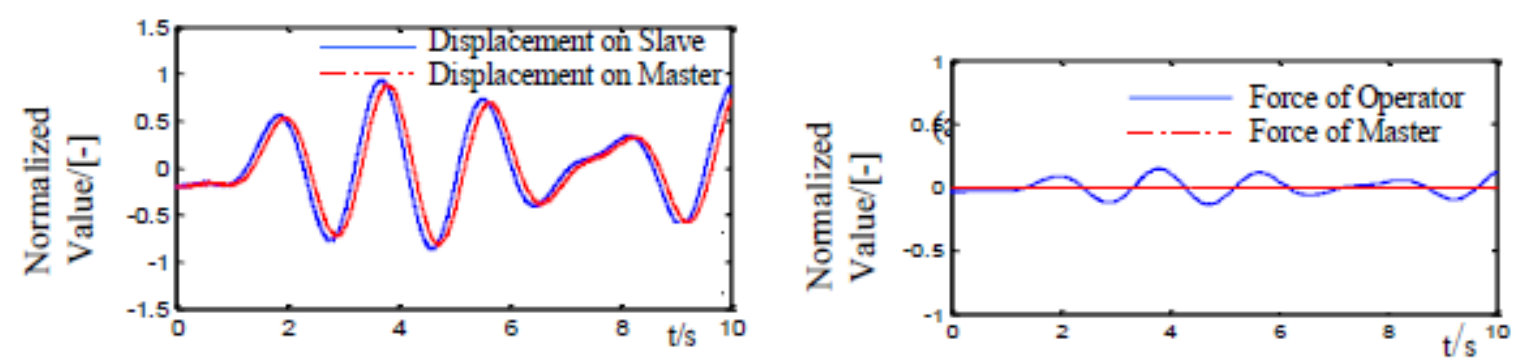

(a) Displacement curve of 1 st cylinder

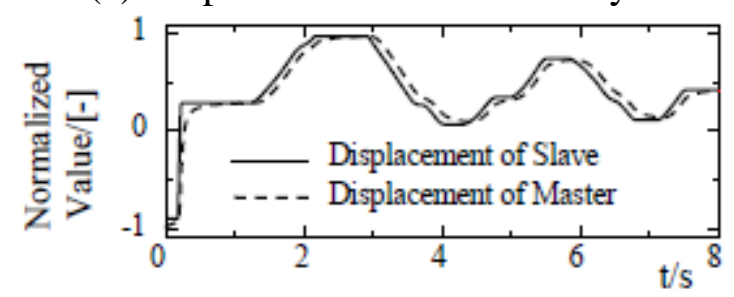

(b) Feedback-force curve of 1st cylinder

(c) Displacement curve of 3rd cylinder

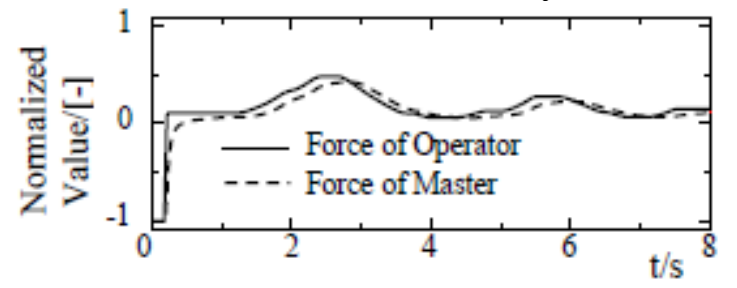

(d) Feedback-force curve of 3rd cylinder

Figure 3. Test curves of the proposed force-feedback.

In terms of the position tracing, the directions of control force and the displacement of the master and slave levers are the same, which ensures a relatively small position tracking error. Phases shown in the figure represent, in turn, the main manipulator force, the slave-side manipulator displacement, the slave-side manipulator force and the main manipulator displacement. When the third cylinder is blocked by the rigid obstacle, the operator would feel the sudden feedback force in the third cylinder of the master-side lever, but no feedback force would be felt in position of the first cylinder. The feedback force increases, but the main manipulator wouldn't move forward as the operator continues applying thrust to the handle. With the novel force-feedback control strategy, the 
operator could not feel the existence of the feedback force from the first cylinder throughout the whole operation, which proves the strategy effective.

\section{Conclusions}

When the slave side interferes with the obstacle in any freedom, the master-side lever generates the same position space with the slave side, helping the operators with better perception of the elements of the slave side interference. The method includes the value and direction of the interference and the information of freedom with interference, which helps the operator master the situation of the slave-side interference better. The experiment results prove the effectiveness of the proposed control strategy in improving the position tracing and force-feedback performances.

\section{Acknowledgment}

The authors are grateful for partial support from Specialized Research Fund for the Doctoral Program of Higher Education of China under Grant No. 20130061110009 and the National Natural Science Foundation of China under Grant No.51305153.

\section{References}

1. H. Li, K. Kawashima, Robot Cim-int. Manuf. 37, 188-196 (2016)

2. K. Mohammadi, H.A. Talebi, M. Zareinejad, Comput. Electr. Eng. 000, 1-12 (2016)

3. D.A. Lawrence, IEEE Trans. Robotic. Autom. 9 (5), 624-637 (1993)

4. S. Kudomi, H. Yamada, T. Muto, JRM 15 (4), 391-397 (2003)

5. DX Zhao, SZ Feng, MD Gong, L Deng, J. JLU (Eng. Tech. Ed.) 38(3), 575-0579 (2008)

6. T. Hirabayashi, J. Akizono, T. Yamamoto, H Sakai, H. Yano, Automat. Constr., 15(5), 563-570 (2006).

7. R. Seifabadi, S. M. Rezaei, S, S, Ghidary, M. Zareinejad, Int. J. Control Autom (IJCAM), 11(4), 768-775 (2013) 